\title{
FAULT-TOLERANT STABILIZATION IN DISCRETE-TIME MULTIPLE-INTEGRATOR NETWORKS WITH GENERAL INFORMATION SHARING
}

\author{
A. Locatelli, N. Schiavoni
}

\begin{abstract}
The paper considers a network of agents with identical discrete-time multiple-integrator dynamics. The agents share information according to an arbitrary topology. The information is relative to the states corresponding to some of the highest integration levels. With reference to this setting, a decentralized stabilization problem is faced, under the further assumption that faults may occur in the communication apparatuses of one or several of the agents. Necessary and sufficient solvability conditions are presented for the problem, together with formulas for a class of least-order regulators.
\end{abstract}

Key Words: Network control, decentralized control, fault-tolerant control, stabilization.

\section{INTRODUCTION}

Networks are systems composed of subsystems, called agents, which share information useful for control. The relevant literature is very vast, and mainly concentrate on identical agents having singleor multiple-integrator dynamics. Most of the papers tackle consensus, synchronization, flocking and similar coordination problems (see, e.g., [1]-[17]). Other papers face stabilization and pole-placement [18]-[20].

Within this general context, we consider a decentralized stabilization problem for a network of discrete-time multiple integrators. We assume that only the states corresponding to one or more of the highest integration levels of the control variables are available for measure. Then, the agents share pieces of this information according to an arbitrary topology. Further, we also include a fault-tolerance condition in the statement [21]. Specifically, we assume that faults may occur in the agents communication apparatuses. In

\footnotetext{
Manuscript received January 24, 2013.

The authors are with the Dipartimento di Elettronica, Informazione e Bioingegneria, Politecnico di Milano, Piazza L. da Vinci, 32, 20133 Milano, Italy \{arturo.locatelli@polimi.it, nicola.schiavoni@polimi.it\}. The corresponding author is N. Schiavoni.
}

particular, we consider the possibility that: (i) one or more agents become unable to transmit information on their states to the other agents; (ii) one or more agents become unable to receive information on the states of the other agents. We require that the closed-loop system remains stable in all these events.

In a continuous-time framework, this problem has already been faced in [22, 23]. The former paper makes reference to double-integrator networks follows the lead of [20], where fault-tolerance is not an explicit issue, and solves a fault-tolerant dominant-pole-placement problem by means of a high-gain regulator. The latter considers multiple-integrator networks, renounces to get pole-placement and proposes low-gain regulators able to supply the system with fault-tolerant stability. In both cases, the exchange of information among the agents pertains only to the states relative to the highestorder integral of the control variables. On the contrary, the lower-order integrals are not available for measure.

Our solution technique has its roots in some previous results of ours, concerning what is called regulator problem in the presence of actuator and sensor faults [24]-[27]. It is not based on generalpurpose techniques for decentralized control design [28]-[30], to be suitably extended to cope with the faulttolerance requirement. Indeed, they require the adoption 
of regulators whose dynamics are concentrated in one or in a few nodes of the network. On the contrary, we propose a class of least-order low-gain regulators, whose dynamics are distributed on the network.

A central role in our fault-tolerant technique is played by a necessary and sufficient condition for the existence of a diagonal matrix such that its product with a given matrix is Hurwitz together with all its principal submatrices [31]. This condition can be considered a nontrivial extension of the classical sufficient condition in [32] for the existence of a diagonal matrix such that its product with a given matrix is Hurwitz (see also $[33,34])$.

In the present discrete-time setting, the solution of a stabilization problem cannot be attained by highgain regulators. Hence, there is no possibility to adopt a strategy similar to the one of [22] in this context. For this reason, reformulating the design technique of [23] to control discrete-time networks is of particular interest. This has already partially been done in [35], where only the highest-order integral of the control variables have been assumed as available for measure. Our aim here is generalizing this result.

By following the line of [23], [35], we tackle the decentralized fault-tolerant stabilization problem outlined above, under the assumption that the states corresponding to some (typically more than one) of the highest integration levels are available for measure. The necessary and sufficient solvability condition is the same as that in [35], but the regulators turn out to be of lower order. Indeed, the regulator order decreases as the number of the states available for measure increases.

The paper is organized as follows. We formally state the fault-tolerant stabilization problem in the next section. Then, we solve it in Section III, after reformulating it in a more convenient way. Subsequently, we illustrate the design technique in Section IV by means of an example. Finally, Section $\mathrm{V}$ contains some concluding remarks. The proofs of the main results are collected in the Appendix.

Notation. Let $\emptyset$ denote the empty set, and, for any set $\vartheta$, let $\kappa_{\vartheta}$ be its cardinality. For any $\sigma \times \sigma$ matrix $K$, let $k_{i, j}$ be its $(i, j)$ element. If $K$ is diagonal, the elements $k_{i, i}$ are denoted by $k_{i}$ for simplicity. For any two sets of integers

$$
\begin{aligned}
& \vartheta_{1}:=\left\{\vartheta_{1 i} \mid 0<\vartheta_{1 i} \leq \sigma \text { and } \vartheta_{1 i} \neq \vartheta_{1 j}, \forall i \neq j\right\} \\
& \vartheta_{2}:=\left\{\vartheta_{2 i} \mid 0<\vartheta_{2 i} \leq \sigma \text { and } \vartheta_{2 i} \neq \vartheta_{2 j}, \forall i \neq j\right\}
\end{aligned}
$$

let

$$
\begin{gathered}
K_{\left[\vartheta_{1}, \vartheta_{2}\right]}:=\left\{k_{\left[\vartheta_{1}, \vartheta_{2}\right] i, j}\right\} \\
k_{\left[\vartheta_{1}, \vartheta_{2}\right] i, j}:=\left\{\begin{array}{cll}
0 & , & j \in \vartheta_{1} \\
0 & , & i \in \vartheta_{2} \\
k_{i, j} & , & \text { otherwise }
\end{array}\right.
\end{gathered}
$$

Matrix $K_{\left[\vartheta_{1}, \vartheta_{2}\right]}$ is $\sigma \times \sigma$. Of course, $K_{[\emptyset, \emptyset]}=K$. For any set of integers $\vartheta$, of the same form as $\vartheta_{1}$ and $\vartheta_{2}$ above, let $K_{(\vartheta)}$ denote the $\left(\sigma-\kappa_{\vartheta}\right) \times\left(\sigma-\kappa_{\vartheta}\right)$ matrix obtained from $K$ after removing its rows and columns with indices in the set $\vartheta$. Conventionally, if $\vartheta=\emptyset$ no row or column is removed. Analogously, for any scalar variable $w$, let's adopt the notations $K_{\left[\vartheta_{1}, \vartheta_{2}\right]}(w)$ and $K_{(\vartheta)}(w)$ for a matrix function $K(w)$. As usual, $I_{\sigma}$ denotes the $\sigma \times \sigma$ identity matrix. Finally, let $o(\zeta)$ be any function such that $\lim _{\zeta \rightarrow 0} O(\zeta) / \zeta=0$

\section{PROBLEM STATEMENT}

We consider a network of $m$ identical agents, whose internal dynamics are constituted by chaines of $n$ discrete-time integrators. As a whole, it is described in the time-domain by

$$
\begin{gathered}
x_{1}(k+1)=x_{1}(k)+x_{2}(k) \\
x_{2}(k+1)=x_{2}(k)+x_{3}(k) \\
\vdots \\
x_{n-1}(k+1)=x_{n-1}(k)+x_{n}(k) \\
x_{n}(k+1)=x_{n}(k)+u(k)
\end{gathered}
$$

Here $u(k)$ and $x_{j}(k), j \in \mathcal{N}:=\{1,2, \ldots, n\}$, are $m$-dimensional vectors: $u(k)$ collects all the control variables, $x_{j}(k)$ collects the $j$-th state variables of all the agents. Of course, $x_{j}(k)$ is the $(n-j+1)$-th (discretetime) integral of $u(k)$.

We assume that only a partial information on the state of the network is available to each agent. Specifically, the $i$-th agent knows $p \leq n$ linear combinations of the state of the network. Each one of them concerns only $x_{j}(k), j \in \mathcal{P}:=\{1,2, \ldots, p\}$, that is, the $p$ highest-order integrals of $u(k)$. The coefficients of these combinations are the same for all $j \in \mathcal{P}$.

Thus, the flow of information throughout the network is described by

$$
y_{j}(k)=G x_{j}(k), j \in \mathcal{P},
$$

where the $y_{j}(k) \mathrm{s}$ are $m$-dimensional vectors, and the $m \times m$ matrix $G:=\left\{g_{i, h}\right\}$ is real and may be referred to as the topology matrix. 
Of course, the $i$-th component of $u(k)$ is the input of the $i$-th agent, whereas the $i$-th components of $y_{j}(k)$, $j \in \mathcal{P}$, are its output.

The model specified by (1), (2) generalizes those frequently adopted in the literature. See, for instance, [20], [23], where $n=2$ and $p=1$, and [23], [35], where $n$ is arbitrary and again $p=1$. In the complex variable domain it can be given the form

$$
Y_{j}(z)=\frac{1}{(z-1)^{n-j+1}} G U(z), j \in \mathcal{P},
$$

where $Y_{j}(z)$ and $U(z)$ are the $Z$-transforms of $y_{j}(k)$ and $u(k)$.

We assume that faults may occur in the communication apparatuses of the agents. Specifically, we suppose that an arbitrary set of agents may become unable to transmit the information about their states to the other agents, and denote this set by $f_{T} \subset \mathcal{M}$. Similarly, we suppose that an arbitrary set of agents may become unable to receive the information about the states of the other agents and denote this set by $f_{R} \subset \mathcal{M}$.

Hence, all the operating conditions of the network are described by the pairs $\left(f_{T}, f_{R}\right) \subset \mathcal{M} \times$ $\mathcal{M}$. Of course, $\left(f_{T}, f_{R}\right)=(\emptyset, \emptyset)$ represents the nominal condition.

Equations (1), (2) or (3) represent the network in nominal conditions. Nominal and faulty conditions can be considered at the same time by substituting (3) with

$$
\begin{gathered}
Y_{j}(z)=\frac{1}{(z-1)^{n-j+1}} G_{\left[f_{T}, f_{R}\right]} U(z), \\
\left(f_{T}, f_{R}\right) \subset \mathcal{M} \times \mathcal{M}, j \in \mathcal{P} .
\end{gathered}
$$

Our aim is controlling the above network by means of a decentralized regulator. In other words, we are interested in regulators composed of $m$ local elements, the $i$-th of which is applied to the $i$-th agent. These regulators are described by

$$
\begin{gathered}
U(z)=\sum_{j \in \mathcal{P}} R_{j}(z) Y_{j}(z), \\
R_{j}(z):=D(z)^{-1} N_{j}(z), \\
N_{j}(z):=\operatorname{diag}\left\{n_{j 1}(z), n_{j 2}(z), \ldots, n_{j m}(z)\right\}, \\
D(z):=\operatorname{diag}\left\{d_{1}(z), d_{2}(z), \ldots, d_{m}(z)\right\},
\end{gathered}
$$

where $R_{j}(z), j \in \mathcal{P}$, is proper, the $1+p$ polynomials $d_{i}(z), n_{1 i}(z), n_{2 i}(z), \ldots, n_{p i}(z)$ do not have common factors, and the $d_{i}(z) \mathrm{s}$ are monic, $i \in \mathcal{M}$.

The loop transfer function of the positive feedback system (3), (5a), (5b) obtained by cutting the loop at $U(z)$, is

$$
L(z):=\sum_{j \in \mathcal{P}} R_{j}(z) \frac{1}{(z-1)^{n-j+1}} G=D_{L}(z)^{-1} N_{L}(z),
$$

where

$$
\begin{gathered}
N_{L}(z):=\sum_{j \in \mathcal{P}}(z-1)^{j-1} N_{j}(z) G, \\
D_{L}(z):=(z-1)^{n} D(z) .
\end{gathered}
$$

Then, by letting

$$
Q(z):=D_{L}(z)-N_{L}(z)
$$

for any $\left(f_{T}, f_{R}\right) \subset \mathcal{M} \times \mathcal{M}$, the characteristic equation of the positive feedback system (4), (5a), (5b), is

$$
\operatorname{det}\left(Q_{\left[f_{T}, f_{R}\right]}(z)\right)=0 \text {. }
$$

Now, we are in the right position to state our stabilization problem.

Problem 1 Find a regulator $R(z)$, of the form (5b)(5d), such that, for all $\left(f_{T}, f_{R}\right) \subset \mathcal{M} \times \mathcal{M}$, system (4), (5a) is stable, that is, all the roots of the characteristic equation (7) lie inside the open unit disk.

Notice that we require that the network (3), when controlled by the decentralized regulator (5), is stable whichever the sets of agents unable to transmit and/or to receive information on their states may be. Hence, a regulator solving Problem 1 actually supplies the control system (3), (5a) with a real fault-tolerance property.

Problem 1 is a generalization of the problem dealt with in [35], where only the case $p=1$ is considered.

\section{PROBLEM REFORMULATION AND SOLUTION}

We now introduce the new Problem 2, and state its equivalence with Problem 1 (Lemma 1). Then, we study the solvability of Problem 2 (Lemmas 2-4). Finally, we give a necessary and sufficient condition for the solvability of Problem 1 and supply explicit formulas for the regulator (Theorem 1). 


\subsection{Problem reformulation}

First, we observe that, for any couple $\left(f_{T}, f_{R}\right) \subset$ $\mathcal{M} \times \mathcal{M}$

$$
\operatorname{det}\left(Q_{\left[f_{T}, f_{R}\right]}(z)\right)=\chi\left(z, f_{T}, f_{R}\right) \prod_{i \in f_{T} \cup f_{R}} \omega_{i}(z)
$$

where

$$
\begin{gathered}
\chi\left(z, f_{T}, f_{R}\right):=\operatorname{det}\left(Q_{\left(f_{T} \cup f_{R}\right)}(z)\right) \\
\omega_{i}(z):=Q_{(\mathcal{M} \backslash\{i\})}(z)= \\
=(z-1)^{n} d_{i}(z)-\sum_{j \in \mathcal{P}}(z-1)^{j-1} n_{j i}(z) g_{i, i}, \\
i \in f_{T} \cup f_{R} .
\end{gathered}
$$

Equations (8)-(9) can easily be proved by recalling (6) and the zero-non-zero pattern of $G_{\left[f_{T}, f_{R}\right]}$. Hence, the characteristic equation (7) of system (4), (5) is equivalent to the set of equations

$$
\begin{gathered}
\chi\left(z, f_{T}, f_{R}\right)=0, \\
\omega_{i}(z)=0, i \in f_{T} \cup f_{R} .
\end{gathered}
$$

Equation (10) is the characteristic equation of the controlled subnetwork composed of the agents not involved in faults. Further, (11) are the characteristic equations of the single controlled agents whose transmitting and/or receiving apparatuses are faulty.

Now, for any given $f \subset \mathcal{M}$, we define the equation

$$
\operatorname{det}\left(Q_{(f)}(z)\right)=0 \text {. }
$$

Then, we consider the following problem.

Problem 2 Find a regulator $R(z)$, of the form (5b)(5d), such that, for all $f \subset \mathcal{M}$, the roots of (12) lie inside the open unit disk.

The relationships between Problems 1 and 2 are very tight. Clearly, (10) and (11) of Problem 1 reduce to (12) of Problem 2 for $f=f_{T} \cup f_{R}$ and $f=\mathcal{M} \backslash\{i\}$, $i \in f_{T} \cup f_{R}$, respectively. Viceversa, (12) of Problem 2 reduces to (10) of Problem 1 if $f_{T} \cup f_{R}=f$. Hence, these observations directly lead us to the following lemma.

Lemma $1 A$ regulator $R(z)$, of the form (5b)-(5d), solves Problem 1 if and only if it solves Problem 2.

\subsection{Problem solution}

We observe that a regulator $R(z)$ can solve the Problem 2 only if the order of each $R_{i}(z)$ is at least $n-p$. Indeed, consider the case $f=\mathcal{M} \backslash\{i\}$. Then, (12) becomes $\omega_{i}(z)=0$. A necessary condition for this equation to have all roots lying inside the open unit disk is that all the roots of the equation

$$
\begin{gathered}
\bar{\omega}_{i}(s):=\omega_{i}(s+1):= \\
=s^{n} d_{i}(s+1)-\sum_{j \in \mathcal{P}} s^{j-1} n_{j i}(s+1) g_{i, i}=0
\end{gathered}
$$

have negative real part. However, if the degree of $d_{i}(z)$ is less than $n-p$, this is impossible, because the coefficient of $s^{n-p}$ in $\bar{\omega}_{i}(s)$ is 0 .

In view of the results in [28], the network is stabilizable for any single $f \subset \mathcal{M}$ by means of a decentralized regulator tuned to that $f$ if and only if $G_{(f)}$ is nonsingular, which means that the network has no fixed modes (at $z=1$ ). However, Problem 2 calls for a single decentralized regulator which stabilizes the network for all $f \subset \mathcal{M}$. Hence, we expect tighter solvability conditions for it.

A first result is the necessary condition given in the forthcoming lemma, where we make use of the matrix $\widetilde{G}$ which retains only the diagonal entries of $G$, namely,

$$
\widetilde{G}:=G_{[\mathcal{M}, \mathcal{M}]}=\operatorname{diag}\left\{g_{1,1}, g_{2,2}, \ldots, g_{m, m}\right\} \text {. }
$$

Lemma 2 Problem 2 admits a solution only if

$$
\operatorname{det}\left(G_{(f)}\right) \operatorname{det}\left(\widetilde{G}_{(f)}\right)>0, \forall f \subset \mathcal{M} \text {. }
$$

Now, we tackle the sufficiency issue. The first step is constituted by Lemma 3. It has its roots in our results in $[25,27]$, and supplies an explicit expression of $R(z)$, under the assumption that a suitable diagonal matrix $V$ can be found. To this aim, we define the $p$ following $m \times m$ matrices:

$$
\begin{aligned}
\Phi_{j}(\varepsilon) & :=\left(\varepsilon^{\frac{(n-j+1)(n-j+2)}{2}}\right) I_{m}, j \in \mathcal{P} \backslash\{p\}, \quad(15 \mathrm{a}) \\
\Psi(z, \varepsilon) & :=\left(\frac{1}{z^{n-p}} \sum_{j=1}^{n-p+1}(z-1)^{n-p+1-j} \varepsilon^{\frac{j(j+1)}{2}}\right) I_{m} .
\end{aligned}
$$

Lemma 3 Problem 2 admits a solution if there exists an $m \times m$ real diagonal matrix $V$ such that, for all $f \subset \mathcal{M}$, the matrices $G_{(f)} V_{(f)}$ are Hurwitz. 
Further, under this condition, there exists $\bar{\varepsilon}>0$ such that, for all $\varepsilon \in(0, \bar{\varepsilon})$, the regulator

$$
R(z):=V\left[\begin{array}{lllll}
\Phi_{1}(\varepsilon) & \Phi_{2}(\varepsilon) & \cdots & \Phi_{p-1}(\varepsilon) & \Psi(z, \varepsilon)
\end{array}\right]
$$

solves Problem 2.

The regulator $R(z)$ of the lemma is actually decentralized, since $V$, the $\Phi_{j}(\varepsilon) \mathrm{s}$ and $\Psi(z, \varepsilon)$ are diagonal. Further, the regulator is low-gain, in the sense that its d.c. gain goes to zero together with $\varepsilon$. The order of the local regulators $R_{i}(z)$ is $n-p$ which, as already noticed, is the least one for Problem 2 to admit a solution. In particular, the regulator dynamics are concentrated on the transfer function $\Psi(z, \varepsilon)$ between $Y_{p}(z)$ and $U(z)$, whereas the transfer functions between $Y_{j}(z), j \in \mathcal{P} \backslash\{p\}$, and $U(z)$ are nondynamic, as given by $\Phi_{j}(\varepsilon)$. Further, trivial, but cumbersome, computations show that the transfer function (15b) can be written as

$$
\Psi(z, \varepsilon):=\left(\frac{\varepsilon}{z^{n-p}} \prod_{j=2}^{n-p+1}\left(z-1+\varepsilon^{j}+o\left(\varepsilon^{j}\right)\right)\right) V .
$$

Thus, $\Psi(z, \varepsilon)$ has $m(n-p)$ finite real transmission zeros. They are located inside the open unit disk, and the distances from $z=1$ of $m$ of them have order of magnitude $\varepsilon^{j}, j=2,3, \ldots, n-p+1$.

Lastly, two extreme, yet meaningful, cases are worth considering. The first is $p=n$, which means that all the state variables are available for measure. In this case, the function (15b) becomes

$$
\Psi(z, \varepsilon)=\varepsilon I_{m},
$$

so that the regulator (16) is nondynamic. The second case is $p=1$, which means only $x_{1}(k)$ is available for measure. The corresponding regulator is

$$
R(z)=\left(\frac{1}{z^{n-1}} \sum_{j=1}^{n}(z-1)^{n-j} \varepsilon^{\frac{j(j+1)}{2}}\right) V .
$$

The importance of Lemma 3 is strictly related to the subproblem of the existence and actual determination of matrix $V$. Some particular cases have been dealt with in [36], where it has been shown that if $G$ is triangular (besides being nonsingular) or else diagonally dominant, then it can simply be set $v_{i}:=$ $-g_{i, i} /\left|g_{i, i}\right|$.

A much more powerful result can be stated by a direct application of a general algebraic theorem of ours [31], concerning what is called stabilization by scaling (see [32]-[34]). Here, we specialize it in the following lemma, with specific reference to the case at hand. Its proof is given in [23].
Lemma 4 An $m \times m$ real diagonal matrix $V$ there exists such that, for all $f \subset \mathcal{M}$, the matrices $G_{(f)} V_{(f)}$ are Hurwitz if and only if (14) holds.

Further, if (14) holds, then there exists $\bar{\tau}>0$ such that, for all $f \subset \mathcal{M}$ and for all $\tau \in(0, \bar{\tau})$, the matrices $-G_{(f)} \widetilde{G}_{(f)} \mathrm{T}_{(f)}(\tau)$, where

$$
\mathrm{T}(\tau):=\operatorname{diag}\left\{\tau, \tau^{2}, \ldots, \tau^{m}\right\},
$$

have distinct real negative eigenvalues.

It is now clear that, for any $\tau \in(0, \bar{\tau})$, the matrix

$$
V:=-\widetilde{G} \mathrm{~T}(\tau)
$$

has the properties required in Lemma 3. As a consequence, the necessary condition (14) is also sufficient for the existence of a solution to Problem 2. Thus, the combination of Lemmas 1-4 supplies the proof of our main result, stated in the following theorem.

Theorem 1 Problem 1 admits a solution if and only if

$$
\operatorname{det}\left(G_{(f)}\right) \operatorname{det}\left(\widetilde{G}_{(f)}\right)>0, \forall f \subset \mathcal{M} .
$$

Further, under this condition there exist $\bar{\tau}>0$ and $\bar{\varepsilon}(\cdot)>0$ such that, for all $\tau \in(0, \bar{\tau})$ and for all $\varepsilon \in$ $(0, \bar{\varepsilon}(\tau))$, the regulator (13), (15)-(18) solves Problem 1 .

It is worth noticing that the necessary and sufficient solvability condition (19) depends only on the topology matrix $G$. On the contrary, it is independend of $n$ and $p$, and in fact it coincides with the condition given in [35] for the case $p=1$. However, the regulator (13), (15)-(18) depends on $p$, and its order decreases when $p$ increases. For any fixed $p$, the order is the least possible one.

Further, we observe that, for all $\left(f_{T}, f_{R}\right) \subset \mathcal{M} \times$ $\mathcal{M}$, the closed-loop system (4), (5a), (13), (15)-(18) enjoys a generalized form of the unconditional stability property, defined in [37], in the sense that any reduction of $\varepsilon$ with respect to a given value belonging to the interval $(0, \bar{\varepsilon}(\tau)), \tau \in(0, \bar{\tau})$, determines a new $R(z)$ which still solves Problem 1.

We finally point out that the regulator (13), (15)(18) solving Problem 1 can be designed according to the following two-step procedure: (i) find a sufficiently small positive $\tau$ such that the matrices $-G_{(f)} \widetilde{G}_{(f)} \mathrm{T}_{(f)}(\tau), f \subset \mathcal{M}$, are Hurwitz (its existence is guaranteed by Lemma 4); (ii) find a sufficiently small positive $\varepsilon$ such that all the roots of (12), $f \subset \mathcal{M}$, lie inside the open unit disk (its existence is guaranteed by Lemma 3). 
Table 1. The magnitudes of the eigenvalues of the controlled network when $p=1$.

\begin{tabular}{|c||c||c||c||c|}
\hline & $\begin{array}{c}f_{T}=\emptyset \\
f_{R}=\emptyset\end{array}$ & $\begin{array}{c}f_{T}=\{1\} \\
f_{R}=\{1\}\end{array}$ & $\begin{array}{c}f_{T}=\{2\} \\
f_{R}=\{4\}\end{array}$ & $\begin{array}{c}f_{T}=\{2,3\} \\
f_{R}=\{2,3\}\end{array}$ \\
\hline$\left|\lambda_{1}\right|$ & 0.2074 & 0.5115 & 0.7036 & 0.5745 \\
$\left|\lambda_{2}\right|$ & 0.7036 & 0.5115 & 0.7036 & 0.5745 \\
$\left|\lambda_{3}\right|$ & 0.7036 & 0.7036 & 0.7036 & 0.7036 \\
$\left|\lambda_{4}\right|$ & 0.7036 & 0.7036 & 0.7036 & 0.7036 \\
$\left|\lambda_{5}\right|$ & 0.7036 & 0.7036 & 0.7036 & 0.7036 \\
$\left|\lambda_{6}\right|$ & 0.7957 & 0.7036 & 0.7036 & 0.7036 \\
$\left|\lambda_{7}\right|$ & 0.9083 & 0.8534 & 0.7036 & 0.8124 \\
$\left|\lambda_{8}\right|$ & 0.9083 & 0.8534 & 0.7036 & 0.8124 \\
$\left|\lambda_{9}\right|$ & 0.9969 & 0.9969 & 0.9970 & 0.9969 \\
$\left|\lambda_{10}\right|$ & 0.9970 & 0.9970 & 0.9970 & 0.9970 \\
$\left|\lambda_{11}\right|$ & 0.9970 & 0.9970 & 0.9970 & 0.9970 \\
$\left|\lambda_{12}\right|$ & 0.9970 & 0.9970 & 0.9970 & 0.9970 \\
\hline
\end{tabular}

\section{ILLUSTRATIVE EXAMPLE}

In order to illustrate our results, we consider a network where the agents are connected in a cyclic way, that is, each one of them shares information with its two neighbors. In particular, we choose $n=2, m=4$ and the topology matrix

$$
G=\left[\begin{array}{llll}
3 & 1 & 0 & 1 \\
1 & 3 & 1 & 0 \\
0 & 1 & 3 & 1 \\
1 & 0 & 1 & 3
\end{array}\right]
$$

which fulfils condition (19). Then, simple computations show that matrices $-G_{(f)} \widetilde{G}_{(f)} \mathrm{T}_{(f)}(\tau), f \subset \mathcal{M}$, are Hurwitz for all positive $\tau$. We set $\tau=1$.

We first let $p=1$, which leads to a 12-th order controlled network, because each local regulator has order 1. It turns out $\bar{\varepsilon} \simeq 0.065$. Thus, we complete the design by setting $\varepsilon=0.055$. Table 1 collects the magnitudes of the closed-loop system eigenvalues in nominal and in three faulty conditions. Stability is obtained in all cases.

For comparison, we now let $p=2$, which leads to an 8-th order controlled network, since the regulator is nondynamic. It turns out $\bar{\varepsilon} \simeq 0.134$. Thus, we complete the design by setting $\varepsilon=0.13$. Table 2 collects the magnitudes of the closed-loop system eigenvalues in nominal and in the faulty conditions considered before. Again, stability is obtained in all cases. We remark that the magnitudes of the dominant eigenvalues are now significantly less than in the previous case. This outcome corresponds to the fact that the information available to the regulator concerns both the first and double integrals of the control variable.

\section{CONCLUSIONS}

The paper has considered a discrete-time network composed of agents with multiple-integrator internal
Table 2. The magnitudes of the eigenvalues of the controlled network when $p=2$.

\begin{tabular}{|l||c||c||c||c|}
\hline & $\begin{array}{c}f_{T}=\emptyset \\
f_{R}=\emptyset\end{array}$ & $\begin{array}{c}f_{T}=\{1\} \\
f_{R}=\{1\}\end{array}$ & $\begin{array}{c}f_{T}=\{2\} \\
f_{R}=\{4\}\end{array}$ & $\begin{array}{c}f_{T}=\{2,3\} \\
f_{R}=\{2,3\}\end{array}$ \\
\hline$\left|\lambda_{1}\right|$ & 0.1528 & 0.1528 & 0.1528 & 0.1528 \\
$\left|\lambda_{2}\right|$ & 0.1528 & 0.1528 & 0.1528 & 0.1528 \\
$\left|\lambda_{3}\right|$ & 0.6277 & 0.3989 & 0.1528 & 0.2373 \\
$\left|\lambda_{4}\right|$ & 0.9330 & 0.7045 & 0.1528 & 0.5429 \\
$\left|\lambda_{5}\right|$ & 0.9823 & 0.9826 & 0.9828 & 0.9827 \\
$\left|\lambda_{6}\right|$ & 0.9828 & 0.9828 & 0.9828 & 0.9828 \\
$\left|\lambda_{7}\right|$ & 0.9828 & 0.9828 & 0.9828 & 0.9828 \\
$\left|\lambda_{8}\right|$ & 0.9830 & 0.9829 & 0.9828 & 0.9829 \\
\hline
\end{tabular}

dynamics, which share information on their states according to an arbitrary topology. Specifically, the information pertains to some of the highest integrals of the control variable. The network had to be controlled by a decentralized regulator. The design objective consisted in stabilizing the closed-loop system in a fault-tolerant fashion, that is, even in the presence of faults in the communication apparatuses of some agents. Within this frame, we have derived a necessary and sufficient solvability condition as well as explicit formulas for a class of least-order regulators.

Among the possible matter for future work, we mention the following issues: (i) exploring the possibility of optimizing the network dynamic behavior, in some sense to be specified; (ii) extending the presented results to the case where the agents have internal dynamics different from that considered here, typically with eigenvalues on arbitrary points of the boundary of the unit circle; (iii) applying the here approach to consensus problems.

\section{Appendix: Proofs}

\section{Proof of Lemma 2.}

Assume that $R(z)$ solves Problem 2, so that all the roots of (12), $f \subset \mathcal{M}$, lie inside the open unit disk. Hence, since $d_{i}(z), i \in \mathcal{M}$, is monic, then $\operatorname{det}\left(Q_{(f)}(z)\right)$ is monic, and it is necessary that (see [38], pp. 97, 98)

$$
\begin{gathered}
\operatorname{det}\left(Q_{(f)}(1)\right)= \\
=\operatorname{det}\left(G_{(f)}\right) \prod_{i \in \mathcal{M} \backslash\{f\}}\left(-n_{1 i}(1)\right)>0, \forall f \subset \mathcal{M} .
\end{gathered}
$$

Consider now the particular case where $f:=\mathcal{M} \backslash\{i\}$, $i \in \mathcal{M}$. Then, condition (20) becomes

$$
-g_{i, i} n_{1 i}(1)>0, \forall i \in \mathcal{M}
$$


which means that $g_{i, i}$ and $n_{1 i}(1)$ have opposite signs. Hence, (20), (21) supply

$$
\operatorname{det}\left(G_{(f)}\right) \prod_{i \in \mathcal{M} \backslash\{f\}} g_{i, i}>0, \forall f \subset \mathcal{M},
$$

which coincides with (14) in view of (13).

\section{Proof of Lemma 3.}

Assume that there exists $V$ such that $G_{(f)} V_{(f)}$ is Hurwitz, $f \subset \mathcal{M}$, and define $R(z)$ according to (13), (15)-(18). The proof consists in showing that the roots of (12), $f \subset \mathcal{M}$, are all inside the open unit disk when $\varepsilon$ is positive small.

Let define $\quad \alpha(f):=m-\kappa_{f} \quad$ and $\quad \mathrm{A}(f):=$ $\{1,2, \ldots, \alpha(f)\}$. Then, trivial, but cumbersome computations show that (12) can be written in the form

$$
\begin{aligned}
\pi(f, z, \varepsilon):= & \sum_{i \in\{0\} \cup \mathrm{A}(f)}(-1)^{i}\left(z^{n-p}(z-1)^{n}\right)^{\alpha(f)-i} \times \\
& \times \beta_{i}(f) r(z, \varepsilon)^{i}=0,
\end{aligned}
$$

where $\beta_{0}(f):=1, \beta_{i}(f)$ is the sum of the principal minors of order $i$ of $G_{(f)} V_{(f)}, i \in \mathrm{A}(f)$, and

$$
\begin{aligned}
r(z, \varepsilon):=z^{n-p} & \sum_{j \in \mathcal{P} \backslash\{p\}}(z-1)^{j-1} \varepsilon^{\frac{(n-j+1)(n-j+2)}{2}}+ \\
& +\sum_{j=1}^{n-p+1}(z-1)^{n-j} \varepsilon^{\frac{j(j+1)}{2}} .
\end{aligned}
$$

Observe that the $\beta_{i}(f)$ s are different from zero, since the $G_{(f)} V_{(f)}$ s are Hurwitz.

For $\varepsilon=0$, (22) has $n \alpha(f)$ roots at $z=1$, the other $(n-p) \alpha(f)$ roots being at $z=0$. Consider the multivalued algebraic function $\gamma(f, \cdot)$, which gives the roots of (22) as functions of $\varepsilon$. By continuity, sufficiency is proved if it is shown that all the branches $\gamma_{\lambda \alpha(f)+h}(f, \cdot), \lambda \in \Lambda:=\{0,1, \ldots, n-1\}, h \in \mathrm{A}(f)$, of $\gamma(f, \cdot)$ starting at $z=1$ move towards the interior of the unit disk for small positive values of $\varepsilon$. Set $z:=$ $s+1$ in (22) and consider the multivalued algebraic function $\bar{\gamma}(f, \cdot)$ which gives the roots of

$$
\pi(f, s+1, \varepsilon)=0
$$

as functions of $\varepsilon$. Then, the branches $\gamma_{\lambda \psi_{f}+h}(f, \cdot)$ move towards the interior of the unit disk for small positive values of $\varepsilon$ if and only if the branches $\bar{\gamma}_{\lambda \psi_{f}+h}(f, \cdot)$ move towards the open left-half of the complex plane for small positive values of $\varepsilon$.
The Newton's polygon (see, for instance, [39, 40]) associated to (23) is drawn in Fig. 1. There, a cross corresponds to any nonzero term of the equation which is relevant to the construction of the diagram. This figure shows that the $n \alpha_{f}$ branches of $\bar{\gamma}(f, \cdot)$ starting at $s=0$ take on the form

$$
\begin{gathered}
\bar{\gamma}_{\lambda \alpha(f)+h}(f, \varepsilon)=\xi_{\lambda h} \varepsilon^{\lambda+1}+o\left(\varepsilon^{\lambda+1}\right), \\
h \in \mathrm{A}(f), \lambda \in \Lambda .
\end{gathered}
$$

In order to find the $\xi_{\lambda h} \mathrm{~s}$, we substitute (24) in (23). By retaining only the dominant terms in $\varepsilon$, we get

$$
\begin{gathered}
\sum_{i \in\{0\} \cup \mathrm{A}(f)}(-1)^{i} \beta_{i}(f) \xi_{0 h}^{\alpha(f)-i}=0, h \in \mathrm{A}(f), \\
\xi_{\lambda h}+1=0, h \in \mathrm{A}(f), \lambda \in \Lambda \backslash\{0\} .
\end{gathered}
$$

Then, (25a) shows that the $\xi_{0 h} \mathrm{~s}$ are the eigenvalues of $G_{(f)} V_{(f)}$, which is a Hurwitz matrix. Hence, the roots (24), $\lambda=0$, move towards the open left-half of the complex plane for small positive $\varepsilon$. Further, in view of (25b), also the roots (24), $\lambda \neq 0$, move towards the open left-half of the complex plane for small positive $\varepsilon$.

The preceding discussion shows that, for any $f \subset$ $\mathcal{M}$, there exists $\bar{\varepsilon}(f)>0$ such that, for all $\varepsilon \in(0, \bar{\varepsilon}(f))$, the roots of (12) lie inside the open unit disk. Then, the lemma follows by letting $\bar{\varepsilon}:=\min _{f \subset \mathcal{M}} \bar{\varepsilon}(f)$.

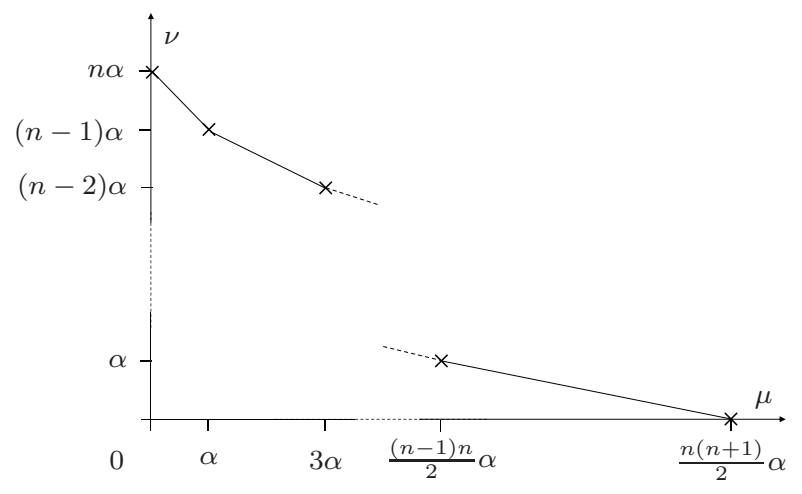

Fig. 1. The Newton polygon associated with the equation (23): $\mu$ is the power of $\varepsilon, \nu$ is the power of $s$ (the dependence of $\alpha$ on $f$ has been dropped).

\section{REFERENCES}

1. Wu, C.W., and L.O. Chua, "Syncronization in an array of linearly coupled dynamical systems", IEEE Trans. Circuits Syst.-I, Vol. 42, pp. 430-447 (1995).

2. Wu, C.W., and L.O. Chua, "Applications of graph theory to the syncronization in an array of coupled nonlinear oscillators", IEEE Trans. Circuits Syst.I, Vol. 42, pp. 494-497 (1995). 
3. Wu, C.W., and L.O. Chua, "Applications of Kroneker products to the analysis of systems with uniform linear coupling", IEEE Trans. Circuits Syst.-I, Vol. 42, pp. 775-778 (1995).

4. Fax, J.A., and R.M. Murray, "Information flow and cooperative control of vehicle formations", IEEE Trans. Automat. Control, Vol. 49, pp. 14651476 (2004).

5. Olfati-Saber, R., "Flocking for multi-agent dynamic systems: algorithms and theory", IEEE Trans. Automat. Control, Vol. 51, pp. 401-420 (2006).

6. Xiao, F., L. Wang and A. Wang, "Consensus problems in discrete-time multiagent systems with fixed topology", J. Math. Anal. Appl., Vol. 322, pp. 587-598 (2006).

7. Olfati-Saber, R., J.A. Fax and R.M. Murray "Consensus and cooperation in networked multiagent systems", Proc. of the IEEE, Vol. 95, pp. 215-233 (2006).

8. Ren, W., "Consensus strategies for cooperative control of vehicle formations", IET Control Theory Appl., Vol. 1, pp. 505-512 (2007).

9. Ren, W., K.L. Moore and Y. Chen, "Highorder and model reference consensus algorithms in cooperative control of multivehicle systems", Trans. ASME, Vol. 129, pp. 678-688 (2007).

10. Ren, W., and E. Atkins, "Distributed multivehicle coordinated control via local information exchange", Int. J. Robust Nonlinear Contr., Vol. 17, pp. 1002-1033 (2007).

11. Roy, S., A. Saberi and K. Herlugson, "A controltheoretic perspective on the design of distributed agreement protocols", Int. J. Robust Nonlinear Contr., Vol. 17, pp. 1034-1066 (2007).

12. Xiao, F., and L. Wang, "Consensus problems for high-dimensional multi-agent systems", IET Control Theory Appl., Vol. 1, pp. 830-837 (2007).

13. Seo, J.H., H. Shim and J. Back, "Consensus of high-order linear systems using dynamic output feedback compensator: low-gain approach", Automatica, Vol. 45, pp. 2659-2664 (2009).

14. Li, Z., D. Zhisheng, and G. Chen, "Consensus of multiagent systems and synchronization of complex networks: a unified viewpoint", IEEE Trans. Circuits Systs. I Reg. Papers, Vol. 57, pp. 213-224 (2010).

15. Jiang, F., and L. Wang, "Consensus seeking of high-order dynamic multi-agent systems with fixed and switching topologies", Int. J. Control, Vol. 83, pp. 404-420 (2010).

16. Yang, T., S. Roy, Y. Wan and A. Saberi, "Constructing consensus controllers for networks with identical general linear agents", Proc. AIAA Guid., Navig., and Contr. Conf., Toronto, Ontario, Canada, pp. 1-22 (2011).

17. Roy, S., Y. Wan, A. Saberi and M. Xue, "Designing linear distributed algorithms with memory for fast convergence", Int. J. Robust Nonlinear Contr., Vol. 22, pp. 1691-1702 (2012).

18. Corfmat, J.P., and A.S. Morse, "Stabilization with decentralized feedback control", IEEE Trans. Automat. Control, Vol. 18, pp. 679-682 (1973).

19. Duan, Z., J. Wang, G. Chen and L. Huang, "Stability analysis and decentralized control of a class of complex dynamical networks", Automatica, Vol. 44, pp. 1028-1035 (2008).

20. Wan, Y., S. Roy, A. Saberi and A. Stoorvogel, "The design of multi-lead-compensator for stabilization and pole placement in double-integrator networks", IEEE Trans. Automat. Control, Vol. 55, pp. 2870-2875 (2010).

21. Blanke, M., M. Kinnaert, J. Lunze and M. Staroswiecki, Diagnosis and Fault-Tolerant Control, Springer-Verlag (2003).

22. Locatelli, A., and N. Schiavoni, "Fault-tolerant pole-placement in double-integrator networks", IEEE Trans. Automat. Control, Vol. 57, pp. 29122917 (2012).

23. Locatelli, A., and N. Schiavoni, "Fault-tolerant stabilization in double-integrator networks", Int. J. Control, Vol. 85, pp. 1663-1670 (2012).

24. Locatelli, A., and N. Schiavoni, "Reliable regulation in centralized control systems", Automatica, Vol. 45, pp. 2673-2677 (2009).

25. Locatelli, A., and N. Schiavoni, 'Fault hiding and reliable regulation in control systems subject to polynomial exogenous signals", Europ. J. Control, Vol. 4, pp. 389-400 (2010).

26. Locatelli, A., and N. Schiavoni, "Reliable regulation by high-gain feedback", Proc. 18th Med. Conf. Contr. Autom., Marrakech, Morocco, pp. 1049-1054 (2010).

27. Locatelli, A., and N. Schiavoni, "Reliable regulation in decentralized control systems", Int. J. Control, Vol. 84, pp. 574-583 (2011).

28. Wang, S., and E. J. Davison, "On the stabilization of decentralized control systems", IEEE Trans. Automat. Control, Vol. 18, pp. 473-478 (1973).

29. Corfmat, J.P., and A.S. Morse, "Decentralized control of linear multivariable systems", Automatica, Vol. 12, pp. 479-495 (1976).

30. Šiljac, D., Decentralized control of complex systems, Academic Press (1994).

31. Locatelli, A., and N. Schiavoni, "A necessary and sufficient condition for the stabilisation of a matrix 
and its principal submatrices", Linear Algebra Appl., Vol. 436, pp. 2311-2314 (2012).

32. Fisher, M.E., and A.T. Fuller, "On the stabilization of matrices and the convergence of linear iterative processes", Math. Proc. Cambridge Phil. Soc., Vol. 54, pp. 417-425 (1958).

33. Roy, S., J. Minteer and A. Saberi, "Some new results on stabilization by scaling", Proc. American Contr. Conf., Minneapolis, Minnesota, USA, pp. 5077-5082 (2006).

34. Roy, S., and A. Saberi, "Scaling: a canonical design problem for networks", Int. J. Contr., Vol. 80, pp. 1342-1353 (2007).

35. Locatelli, A., and N. Schiavoni, "Fault-tolerant stabilization in discrete-time multiple-integrator networks", Proc. American Contr. Conf., Montréal, Québec, Canada, pp. 1231-1236 (2012).

36. Locatelli, A., R. Scattolini and N. Schiavoni, "On the design of reliable robust decentralized regulators for linear systems", Large Scale Systems, Vol. 10, pp. 95-113 (1986).

37. Campo, P.J. and M. Morari, "Achievable closedloop properties of systems under decentralized control: conditions involving the steady-state gain”, IEEE Trans. Automat. Control, Vol. 39, pp. 932-943 (1994).

38. Ȧström, K.J. and B. Wittenmark, Computer Controlled Systems: Theory and Design, PrenticeHall (1984).

39. Bliss, G.A., Algebraic Functions, American Mathematical Society (1933).

40. Postletwaite, I. and A.G.J. MacFarlane, A Complex Variable Approach to the Analysis of Linear Multivariable Systems, Springer-Verlag (1979).

\section{AUTHORS' BIOGRAPHIES}

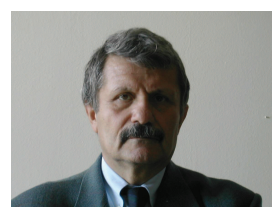

Arturo Locatelli received the Laurea degree in electronic engineering from the Politecnico di Milano, Milano, Italy, in 1964 and the Libera Docenza degree in automatic control in 1971. He was research fellow from 1966 to 1969 and Assistant Professor from 1969 to 1975 at the Politecnico di Milano where he has been Professor of control theory since 1976.



Nicola Schiavoni was born in Manduria, Italy, in 1950 and received the Laurea degree in electronic engineering from the Politecnico di Milano, Milano, Italy, in 1973. Since then, he has been with the Centro di Teoria dei Sistemi of the CNR (the italian national research council) and the Politecnico di Milano, where he holds the position of Full Professor of Automatic Control in the Dipartimento di Elettronica, Informazione e Bioingegneria. 